\title{
Comment
}

\section{Pharmacological attenuation of blood pressure variability}

\author{
Claude JULIEN ${ }^{1}$
}

Laboratoire de Physiologie, Faculté de Pharmacie, Université Claude Bernard Lyon 1, Lyon 69373, France

\author{
${ }^{1}$ Correspondence to Dr Claude JULIEN. \\ Phn 33-478-777072. \\ Fax 33-478-777118. \\ E-mail julien@univ-lyon1.fr \\ Received 2005-08-01 \\ Accepted 2005-09-07 \\ doi: $10.1111 /$ j.1745-7254.2005.00221.x
}

Over the past few years, the research team of Professor Ding-feng SU has reported an impressive quantity of experimental data about the relationships between blood pressure variability (BPV) and end-organ damage, a topic of obvious clinical interest. This research work has been summarized in a paper that appeared in the August issue of the renowned journal Trends in Pharmacological Sciences ${ }^{[1]}$. The studies by $\mathrm{Su}$ et al provide convincing evidence that BPV is an independent cardiovascular risk factor that should be considered as such and, therefore, might become an important target for therapeutic interventions. Besides these exciting perspectives in the prevention and treatment of cardiovascular diseases, the work by Su et al raises a series of physiological questions.

In most, if not all, studies by Su et al, BPV is expressed as the standard deviation of beat-to-beat blood pressure data ${ }^{[2,3]}$. The standard deviation provides an index of overall BPV that incorporates all kinds of blood pressure (BP) variations, from those resulting from the respiratory cycle to the slow trends occurring over the whole recording period. However, when studied in the frequency domain, it becomes apparent that the bulk of BPV is concentrated at low frequencies $(<0.15 \mathrm{~Hz} \text { in rats })^{[4]}$. In other words, the standard deviation of BP essentially corresponds to the low-frequency component of the BP spectra. In the low frequency band, BPV is mainly the result of opposing interactions between hemodynamic perturbations and the corrective feedback provided by the arterial baroreceptor reflex. Hemodynamic studies in conscious rats with neonatal chemical sympathectomy ${ }^{[5]}$, chronic sinoaortic baroreceptor denervation ${ }^{[6]}$, and acute neurohumoral blockade ${ }^{[7]}$ have revealed that one major source of slow (or low frequency) hemodynamic perturbations is the myogenic response of vascular smooth muscle cells that promotes vasoconstriction in regional circulations when $\mathrm{BP}$ increases and vasodilatation when BP decreases (Figure 1) ${ }^{[8]}$.

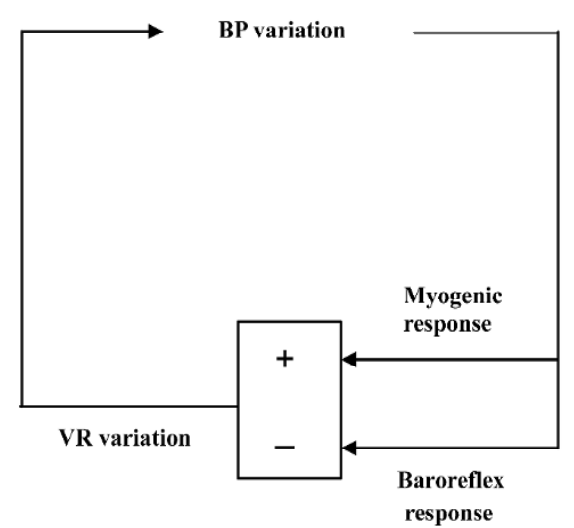

Figure 1. Schematic diagram of opposing interactions between myogenic and baroreflex responses. The relative importance of these responses determines the net change in vascular resistance (VR) in regional vascular beds as well as in the systemic circulation.

Taking into consideration this fundamental underlying physiology of BPV, the soundest pharmacological approach to reducing BPV is twofold. First, it would be beneficial to enhance baroreflex function. Chronic treatment with a nonantihypertensive dose of ketanserin increases baroreflex sensitivity while reducing BPV in spontaneously hypertensive rats ${ }^{[3]}$. This effect was observed for the cardiac component of the baroreceptor reflex. It is not known whether this also applies to the sympathetic component. The second 
approach would be to attenuate myogenic responses of regional circulations (Figure 1). This can be achieved with dihydropyridine L-type calcium channel blockers. Su et al have demonstrated that nitrendipine can selectively reduce $\mathrm{BPV}^{[2]}$. However, this strategy is more problematic than the first because although the reduction of BPV afforded by calcium channel blockers would be beneficial in terms of arterial stiffness and left ventricular workload, this would probably be achieved at the cost of a reduced protection of capillary vascular beds (eg in the brain and kidney) ${ }^{[9]}$. Further investigations including regional hemodynamic measurements are required to clarify this issue. Finally, it should be noted that the research team of Professor Su will soon make available a rat strain with a spontaneous deficiency in baroreflex function ${ }^{[10]}$, which will undoubtedly facilitate the study of pharmacological modulation of BPV.

\section{References}

1 Su DF, Miao CY. Reduction of blood pressure variability: a new strategy for the treatment of hypertension. Trends Pharmacol Sci 2005; 26: 388-90.

2 Liu JG, Xu LP, Chu ZX, Miao CY, Su DF. Contribution of blood pressure variability to the effect of nitrendipine on end-organ damage in spontaneously hypertensive rats. J Hypertens 2003; 21: 1961-7.

3 Xie HH, Shen FM, Cao YB, Li HL, Su DF. Effects of low-dose ketanserin on blood pressure variability, baroreflex sensitivity and end-organ damage in spontaneously hypertensive rats. Clin Sci (Lond) 2005; 108: 547-52.

4 Chapuis B, Vidal-Petiot E, Oréa V, Barrès C, Julien C. Linear modelling analysis of baroreflex control of arterial pressure variability in rats. J Physiol 2004; 559: 639-49.

5 Zhang ZQ, Julien C, Gustin MP, Cerutti C, Barrès C. Hemodynamic analysis of arterial pressure lability in sympathectomized rat. Am J Physiol 1994; 267: H48-56.

6 Zhang ZQ, Barrès C, Julien C. Involvement of vasodilator mechanisms in arterial pressure lability after sino-aortic baroreceptor denervation in rat. J Physiol 1995; 482: 435-48.

7 Létienne R, Barrès C, Cerutti C, Julien C. Short-term haemodynamic variability in the conscious areflexic rat. J Physiol 1998; 506: 263-74.

8 Burattini R, Borgdorff P, Westerhof N. The baroreflex is counteracted by autoregulation, thereby preventing circulatory instability. Exp Physiol 2004; 89: 397-405.

9 Griffin KA, Hacioglu R, Abu-Amarah I, Loutzenhiser R, Williamson GA, Bidani AK. Effects of calcium channel blockers on "dynamic" and "steady-state step" renal autoregulation. Am J Physiol Renal Physiol 2004; 286: F1136-43.

$10 \mathrm{Su}$ DF, Miao CY. Arterial baroreflex function in conscious rats. Acta Pharmacol Sin 2002; 23: 673-9. 